\title{
MINIMAX VARIATIONAL PRINCIPLE FOR STEADY BALANCED SOLUTIONS OF THE ROTATING SHALLOW WATER EQUATIONS*
}

\author{
VISWESWARAN NAGESWARAN ${ }^{\dagger}$ AND BRUCE TURKINGTON TU $^{\ddagger}$ \\ Dedicated to the sixtieth birthday of Professor Andrew Majda
}

\begin{abstract}
Two well-known variational principles for geophysical flows are combined into a single minimax principle that characterizes distinguished steady solutions of the rotating shallow water (RSW) equations. On the one hand, in the limit of small Rossby number $\epsilon$, in which the dynamics becomes quasi-geostrophic and closes terms of the potential vorticity field $Q$, steady coherent states are characterized as minimizers of (generalized) enstrophy $\mathcal{A}$ at a given value of total energy $\mathcal{H}$. On the other hand, for small amplitude motions at finite $\epsilon$ balanced states resulting from geostrophic adjustment are characterized as minimizers of the total energy $\mathcal{H}$ subject to a given potential vorticity $Q$. Moreover, the organization into a coherent state through potential vorticity mixing occurs on a slow time scale relative to the fast time scale of adjustment through inertia-gravity wave radiation. These two complementary principles suggest a variational characterization of steady balanced states for the RSW equations at finite $\epsilon$. Namely, the functional $\mathcal{A}+\theta \mathcal{H}$, where $\theta<0$ is a parameter, is first maximized over all RSW fields with given $Q$, and then minimized over all $Q$. Any such minimax critical point of $\mathcal{A}+\theta \mathcal{H}$ is an exact steady solution of the RSW equations, which represents a physically relevant equilibrium state at finite Rossby number. This minimax principle is implemented numerically for zonal shear flows, and branches of solutions are computed to first-order in $\epsilon$. The results quantify the breakdown of quasi-geostrophy and the asymmetry between cyclonic and anticyclonic structures. In addition, the $O(\epsilon)$-correction is computed for a model of the zonally-averaged winds in Jupiter's weather layer.
\end{abstract}

Key words. Rotating shallow water model, steady flow, variational principle, potential vorticity, geostrophic adjustment.

AMS subject classifications. 76U05, 76B55.

\section{Introduction}

The Rotating Shallow Water (RSW) equations constitute the simplest equations in geophysical fluid dynamics that govern both slow vortical motions and fast inertiagravity waves. While they greatly simplify the vertical structure and thermodynamics of a stratified fluid, they provide a physically realistic model of key phenomena described by the full primitive equations $[20,29,35]$. In the limit of small Rossby number, the RSW equations become the quasi-geostrophic (QG) equations, which close in terms of the potential vorticity (PV) field and filter the inertia-gravity waves. In this limit there is an extensive modern theory of nonlinear coherent structures - organized flows, such as shear layers or vortices, that emerge and persist within geostrophic turbulence. A comprehensive presentation of this theory and its applications is given in the monograph by Majda and Wang [25]. The most complete part of this theory characterizes the statistical equilibrium states of quasi-geostrophic flows. Using the conservation properties of the PV transport equation as a basis, equilibrium states are realized as maximizers of the entropy of PV mixing subject to constraints on total energy and circulation. Such constrained entropy maximizing states are distinguished steady flows in two senses. First, they are most probable states with respect to fine-grained PV fluctuations; second, they are nonlinearly stable states with respect to coarse-grained perturbations $[15,16]$. This modern equilibrium statistical

\footnotetext{
*Received: October 30, 2009; accepted (in revised version): June 15, 2009.

${ }^{\dagger}$ Credit Suisse, New York, NY 10010 (visweswaran.nageswaran@credit-suisse.com).

${ }^{\ddagger}$ Department of Mathematics and Statistics, University of Massachsetts, Amherst, MA 01003 (turk@math.umass.edu).
} 
theory has been successful in predicting the properties of the long-lived, large-scale structures that are observed in geophysical fluid dynamics [9]. For instance, it has been implemented to model the formation of intense jets or spots in the weather layer of Jupiter's atmosphere [4, 33].

The present work is motivated by the desire to extend these mature variational characterizations of coherent structures for the QG equations to the RSW equations. That is, we seek to understand the influence of a small but finite Rossby number on the long-time organization of a turbulent flow. A completely satisfactory statistical equilibrium theory of RSW flows is not yet available, although the maximum entropy formalism has been applied to the RSW system [7, 37]. The fundamental difficulty to be confronted in making the desired extension from QG dynamics to RSW dynamics at finite Rossby number is the presence of inertia-gravity waves and their coupling with vortical motions. At finite Rossby number, there are two distinct physical mechanisms that underlie the organization of the flow into a coherent state. On a fast time scale, the radiation of inertia-gravity waves tends to minimize energy over a fixed PV field, through a well-known mechanism called geostrophic adjustment [20, 34]. On a slow time scale, PV mixing tends to maximize entropy subject to fixed energy $[26,15]$. Our central result is a synthesis of these two extremization principles into a variational characterization of physically relevant steady states of the RSW equations. The distinguished states that we characterize are minimax critical points of a functional constructed from the total energy and a generalized enstrophy, which is identical with a negative entropy in the statistical theory.

Our construction may be outlined as follows.

Let $(u, v)$ be the horizontal velocity field of a RSW flow with free-surface height perturbation $\eta$. In the shallow vertical direction, $z=0$ denotes the undisturbed freesurface, while $z=-H(x, y)$ is the underlying bottom topography. The fluid column height is therefore $H+\eta$. Let $D$ denote the mean depth scale. The RSW equations with constant Coriolis parameter $f$ and gravitational acceleration $g$ are

$$
\begin{aligned}
\frac{D u}{D t}-f v & =-g \frac{\partial \eta}{\partial x} \\
\frac{D v}{D t}+f u & =-g \frac{\partial \eta}{\partial y} \\
\frac{D}{D t}(H+\eta)+(H+\eta)\left(\frac{\partial u}{\partial x}+\frac{\partial v}{\partial y}\right) & =0 .
\end{aligned}
$$

The unknowns $u, v, \eta$ are functions of the horizonal coordinates $(x, y)$ and time $t$; the material derivative is $D / D t=\partial / \partial t+u \partial / \partial x+v \partial / \partial y$. Associated with these equations is the remarkable conservation of potential vorticity, namely,

$$
\frac{D Q}{D t}=0, \quad \text { where } Q=\frac{f+\zeta}{H+\eta}
$$

in which $\zeta=\partial v / \partial x-\partial u / \partial y$ is the relative vorticity. This equation describes the advection of the potential vorticity $Q$ along fluid parcels and puts a strong constraint on the RSW dynamics.

The governing equations have the integral invariants

$$
\mathcal{H}=\frac{1}{2} \int\left[(H+\eta)\left(u^{2}+v^{2}\right)+g \eta^{2}\right] d x d y
$$




$$
\mathcal{A}=\int(H+\eta) a(Q) d x d y .
$$

$\mathcal{H}$ is the total energy, while $\mathcal{A}$ denotes a family of invariants called generalized enstrophies, in which $a(Q)$ is any (sufficiently regular) real function of the PV; the particular case $a=Q^{2} / 2$ is used in most phenomenological studies of turbulence. All other RSW invariants are associated with symmetry under spatial translation or rotation, and they depend upon the invariance of the topography $H$. For the sake of clarity in this introduction, we suppress them here.

We are concerned with regimes of motion in which the Rossby number $\epsilon=U / f L$ is small; $L$ and $U$ denote characteristic horizontal length and velocity scales. In real applications, typically $\epsilon$ is on the order of 0.1 . We are especially interested in extending to finite $\epsilon$ the theory of coherent states for the quasi-geostrophic (QG) dynamics, which pertain to the limit $\epsilon \rightarrow 0$. To motivate our minimax principle, we therefore recall some of the results known in the QG theory. The standard QG limit is obtained for a scaling in which $\lambda=L_{d} / L$ is fixed, where $L_{d}=\sqrt{g D} / f$ is the Rossby deformation scale, and, if bottom topography is present, $H=D-\epsilon b(x, y)$, for a fractional bottom height $b$ [29]. Then, in the singular limit as $\epsilon \rightarrow 0$, the asymptotic dynamics aer governed by the single advection equation

$$
\frac{D q}{D t}=0, \quad \text { where } \quad q=\Delta \psi-\lambda^{-2} \psi+b
$$

in which $q$ is the QG potential vorticity and $\psi$ is the geostrophic streamfunction which determines the velocity field according to $u=-\partial \psi / \partial y, v=\partial \psi / \partial x$. In this asymptotic analysis, the RSW PV is related to the QG PV by $Q=(f / D)\left[1+\epsilon q+O\left(\epsilon^{2}\right)\right]$.

The QG invariants, total energy and generalized enstrophy, take the form

$$
\begin{aligned}
\mathcal{H}_{0} & =\frac{1}{2} \int\left[|\nabla \psi|^{2}+\lambda^{-2} \psi^{2}\right] d x d y \\
\mathcal{A}_{0} & =\int a(q) d x d y
\end{aligned}
$$

In modern statistical equilibrium theory the functional $\mathcal{A}_{0}$ is identified as a negentropy (negative entropy) of fine-scale PV mixing [15]. Associated with any given probability distribution of fine-grained PV fluctuations is a unique convex function $a(q)$ which defines the integrand of the negentropy functional $\mathcal{A}_{0}$. For instance, if the PV fluctuations are Gaussian, then $a(q)$ is quadratic, and $\mathcal{A}_{0}$ coincides with the classical enstrophy of phenomenological turbulence theory. Given a statistical model of fine-grained PV fluctuations, and hence an associated convex function $a(q)$, the most probable coarse-grained $\mathrm{PV}$ field is the minimizer of $\mathcal{A}_{0}$ subject to the energy constraint $\mathcal{H}_{0}=E$ (and a circulation constraint, as appropriate). The resulting minimizer is a steady solution of the QG equations, and it represents the coherent structure that forms and persists within a turbulent PV field.

This modern perspective supercedes earlier approaches in which Gibbsian statistical mechanics was applied to the quadratic invariants that are preserved under spectral truncation of the governing dynamics [30, 36]. In those earlier statistical models, the distributions are Gaussian and the mean-field equations for the coherent states are linear. From the point of view of general equilibrium statistics, the quadratic enstrophy integral is precisely the negentropy of the coarse-grained PV field when a Gaussian prior distribution is imposed on fine-grained PV fluctuations. 
Accordingly, classical theory is just a special case of modern theory. Moreover, the variational principle for equilibrium states in the Gaussian model coincides with the so-called minimum enstrophy principle $[5,23,26]$, which may be motivated by cascade arguments.

With the goal of extending the QG variational principle to finite $\epsilon$, let us instead consider the associated unconstrained variational principle, namely,

$$
\min _{q} \mathcal{A}_{0}(q)+\theta \mathcal{H}_{0}(q)
$$

in which we introduce a Lagrange multiplier $\theta$. When $\mathcal{A}_{0}$ is interpreted as a negentropy, the parameter $\theta$ plays the role of an inverse temperature. Throughout this paper we impose the restriction that the parameter $\theta$ be negative, and we assume that $a$ is strictly convex. For $\theta<0$, the QG variational principle produces isolated coherent structures, such as vortices or free shear layers, which are the most interesting coherent structures in QG turbulence. For $\theta>0$, the structures tend to organize near boundaries.

A minimizer $q$ of $\mathcal{L}_{0}=\mathcal{A}_{0}+\theta \mathcal{H}_{0}$ defines a steady QG flow. This basic fact is readily verified by calculating the first variation

$$
\delta \mathcal{L}_{0}=\int\left[a^{\prime}(q)-\theta \psi\right] \delta q d x d y .
$$

At a minimizer $q, \delta \mathcal{L}_{0}=0$, which implies that there is a functional dependence, $a^{\prime}(q)=$ $\theta \psi$, between PV and streamfunction; hence $\psi$ defines an exact steady QG flow $[15,25]$. Moreover, an analysis of the second variation, $\delta^{2} \mathcal{L}_{0}$, shows that a nondegenerate minimizer is nonlinearly stable, because $\mathcal{L}_{0}$ is an exact conserved quantity of the QG dynamics and hence supplies a Lyapunov functional. This nonlinear stability result is equivalent to the so-called second Arnold stability theorem [15, 25].

With these QG principles in mind, we return to the RSW equations and seek an analogous principle for distinguished steady flows at finite Rossby number. Intuitively, we conceive of relaxation into equilibrium as a two time-scale process: fast geostrophic adjustment coupled with slow PV mixing. For the variational characterization of the fast process, we appeal to the principle articulated by Vallis [34, 35], in which the end state of the geostrophic adjustment process is taken to be the state of "extreme balance" - that is, the energy-minimizing state at fixed $Q$. But Vallis's principle is only an approximation for finite amplitude RSW motions, and so we modify it slightly by including the generalized enstrophy. Namely, we introduce the constrained variational principle

$$
\max _{(u, v, \eta)} \mathcal{A}(u, v, \eta)+\theta \mathcal{H}(u, v, \eta) \quad \text { subject to } \frac{f+\zeta}{H+\eta}=Q .
$$

For given $\theta<0$ and given $Q$, the solution of this maximization problem defines a field $\left(u^{*}, v^{*}, \eta^{*}\right)$ analogous to the extreme balance state.

Our minimax principle for RSW dynamics synthesizes the two variational principles described above. The objective functional is $\mathcal{L}=\mathcal{A}+\theta \mathcal{H}$, a linear combination of exact RSW invariants. The RSW potential vorticity $Q$ is related to the primitive variables $(u, v, \eta)$ pointwise by $\Pi(u, v, \eta)=(f+\zeta) /(H+\eta)$. We propose the minimax variational principle

$$
\min _{Q} \max _{\Pi(u, v, \eta)=Q} \mathcal{L}(u, v, \eta)
$$


in which the inner maximization is over all $\operatorname{RSW}$ fields $(u, v, \eta)$ with a given $Q$ and appropriate boundary conditions (free slip velocity along boundary walls), while the outer minimization is over all $\mathrm{PV}$ fields $Q$. Any such minimax state, being a critical point of $\mathcal{L}$, is an exact steady RSW flow. This fact follows by verifying that the vanishing of the first variation, $\delta \mathcal{L}=0$, implies the RSW steady flow equations. Furthermore, any minimax solution produces a distinguished steady state in the sense that it is optimized with respect to both potential vorticity advection and inertiagravity wave radiation. In essence, the inner maximization realizes fast geostrophic adjustment, while the outer minimization achieves slow PV mixing.

The saddle point nature of these solutions combined with the necessary restriction that $\theta<0$ does not allow us to infer stability by using the exact invariant $\mathcal{L}$ as a Lyapunov functional. Nonetheless, the separation of time scales between fast adjustment and slow PV mixing suggests that these minimax states have special physical significance. Indeed, they represent a natural extension to finite $\epsilon$ of the steady, stable coherent states in the QG theory, to which they tend in the limit $\epsilon \rightarrow 0$.

We refer to these distinguished critical points of $\mathcal{L}$ as steady, balanced solution of the RSW equations, even though the term "balance" is normally understood to pertain to time-varying states that do not excite gravity waves. Indeed, previous investigations of the influence of gravity waves on vortical motions have focused on multiple-scale asymptotics to derive balanced dynamics for the RSW equations $[1,2,3]$, or on direct numerical simulations of RSW flows [8, 17, 22, 28]. To the best of our knowledge a direct characterization of steady balanced flows in the context of RSW equations has not been articulated, and therefore our minimax principle partly fills a lacuna in the literature. An understanding of these distinguished steady states may throw some light on the coupling of inertia-gravity wave activity and potential vorticity mixing, especially over very long time intervals.

The paper is organized as follows. We begin in section 2 by verifying that the minimax critical points are exact steady flows. In section 3 we motivate and formulate our minimax variational principle from the appropriately nondimensionalized RSW equations. In section 4 we solve the inner maximization problem by means of an asymptotic expansion in Rossby number $\epsilon$, thereby obtaining the first-order correction to QG fields for finite $\epsilon$. We implement a numerical optimization method to solve the resulting outer minimization problem. In section 5 we present some computed solutions in the simplest physically relevant setting - namely, zonal shear flows in a channel domain with flat topography. These results quantify the deviation from quasigeostrophy and the cyclone-anticyclone asymmetry of RSW flows as Rossby number is increased. Finally, in section 6 we adapt our method to compute zonal shear flows for the same model of Jupiter's weather layer as was used in the QG model in [33], thereby quantifying the effects of a finite Rossby number on those predictions.

\section{Exact steady RSW flows}

Before proceeding with a detailed physical motivation for our minimax principle (1.1), we establish in this section the fact that any critical point of the functional $\mathcal{L}$ is an exact steady solutions of the RSW equations of motion. In the following section, we explain why the minimax critical points have a special significance, which is related to the notion of balance in asymptotic models.

To do so we calculate the first variations of the functions $\mathcal{A}$ and $\mathcal{H}$ that make up 
the objective functional $\mathcal{L}=\mathcal{A}+\theta \mathcal{H}$. For the generalized enstrophy, we obtain

$$
\begin{aligned}
\delta \mathcal{A} & =\int(H+\eta) a^{\prime}(Q) \delta Q+a(Q) \delta \eta \\
& =\int a^{\prime}(Q) \delta \zeta+\left[a(Q)-Q a^{\prime}(Q)\right] \delta \eta \\
& =\int \frac{\partial}{\partial y} a^{\prime}(Q) \delta u-\frac{\partial}{\partial x} a^{\prime}(Q) \delta v+\left[a(Q)-Q a^{\prime}(Q)\right], \delta \eta
\end{aligned}
$$

using $\delta Q=(H+\eta)^{-1}[\delta \zeta-Q \delta \eta]$, and then $\delta \zeta=\partial(\delta v) / \partial x-\partial(\delta u) / \partial y$. For the energy, we have

$$
\delta \mathcal{H}=\int(H+\eta)(u \delta u+v \delta v)+\left[\frac{1}{2}\left(u^{2}+v^{2}\right)+g \eta\right] \delta \eta
$$

To proceed further with the calculation we introduce the Legendre transform of the convex real function $a(Q)$. Namely, let

$$
\Psi=a^{\prime}(Q), \quad a^{*}(\Psi)=Q \Psi-a(Q)=\max _{\tilde{Q}}[\tilde{Q} \Psi-a(\tilde{Q}),]
$$

where prime denotes $d / d Q$. In terms of $\Psi$ and $a^{*}(\Psi)$, the vanishing of the first variation, $\delta \mathcal{L}=0$, gives the following three equations:

$$
\begin{aligned}
& 0=\frac{\delta \mathcal{L}}{\delta u}=\frac{\partial \Psi}{\partial y}+\theta(H+\eta) u \\
& 0=\frac{\delta \mathcal{L}}{\delta v}=-\frac{\partial \Psi}{\partial x}+\theta(H+\eta) v \\
& 0=\frac{\delta \mathcal{L}}{\delta \eta}=-a^{*}(\Psi)+\theta\left[\frac{1}{2}\left(u^{2}+v^{2}\right)+g \eta\right] .
\end{aligned}
$$

Also, using $(f+\zeta) /(H+\eta)=Q=d a^{*} / d \Psi$, where the second equality expresses the involutive property of the Legendre transform, $(2.3,2.4)$ are equivalent to the pair of equations

$$
\begin{aligned}
-(f+\zeta) v & =-\theta^{-1} \frac{\partial}{\partial x} a^{*}(\Psi) \\
(f+\zeta) u & =-\theta^{-1} \frac{\partial}{\partial y} a^{*}(\Psi) .
\end{aligned}
$$

To verify that a critical point $(u, v, \eta)$ of $\mathcal{L}$ defines a steady solution of the RSW equations, we write the steady flow equations in the form:

$$
\begin{gathered}
-(f+\zeta) v=-\frac{\partial}{\partial x}\left[\frac{1}{2}\left(u^{2}+v^{2}\right)+g \eta\right] \\
(f+\zeta) u=-\frac{\partial}{\partial y}\left[\frac{1}{2}\left(u^{2}+v^{2}\right)+g \eta\right] \\
\frac{\partial}{\partial x}[(H+\eta) u]+\frac{\partial}{\partial y}[(H+\eta) v]=0 .
\end{gathered}
$$

This alternative form of the governing equations displayed in the introduction follows from the vector analysis identity $V \cdot \nabla V=\nabla\left(\frac{1}{2} V^{2}\right)-V \times(\nabla \times V)$. The momentum 
equations follow from $(2.6,2.5)$, and the continuity equation is a consequence of $(2.3$, 2.4). This establishes the claim that every critical point of $\mathcal{L}$ defines an exact steady flow.

In the above derivation of the steady RSW equations from the variational principle we use variations in $u$ and $v$ that are supported in the fluid domain. If we allow general variations $\delta u$ and $\delta v$ satisfying the free slip boundary condition $(\delta u, \delta v) \cdot N=0$, where $N$ is the unit normal to the boundary, then we obtain $a^{\prime}(Q)=0$ on the boundary; this results from a boundary integral term in the integration by parts of the $\delta \mathcal{A}$ expression. This variational boundary condition is equivalent to the usual requirement that $Q=$ const. on the boundary, and the arbitrary constant can be included in the variational formulation by replacing $a(Q)$ with $a(Q)+\alpha Q$ for a constant $\alpha$. In turn, $\alpha$ can be identified with a Lagrange multiplier for a constraint on the total circulation $\mathcal{C}=\int \zeta$.

We remark that the formal Hamiltonian structure of the RSW equations furnishes a quick proof that any solution of the minimax problem (1.1) is an exact steady flow [31]. Indeed, the fact that $\mathcal{H}$ is a Hamiltonian and that all functionals $\mathcal{A}$ are Casimirs implies that for any $\theta \neq 0, \mathcal{H}+\theta^{-1} \mathcal{A}$ is a Hamiltonian. Consequently, all critical points of $\mathcal{L}$ are fixed points of the Hamiltonian dynamics.

\section{Motivation of the minimax principle}

In nondimensionalized form, the Rotating Shallow Water equations are

$$
\begin{gathered}
\epsilon \frac{D u}{D t}-v=-\frac{\partial \eta}{\partial x}, \quad \epsilon \frac{D v}{D t}+u=-\frac{\partial \eta}{\partial y} \\
\epsilon \frac{D}{D t}\left(\lambda^{-2} \eta-b\right)+\left[1+\epsilon\left(\lambda^{-2} \eta-b\right)\right]\left(\frac{\partial u}{\partial x}+\frac{\partial v}{\partial y}\right)=0
\end{gathered}
$$

where $u$ and $v$ represent the velocities in the $x$ and $y$ directions respectively, $\eta$ the free surface elevation, and $b$ the scaled bottom topography. The dimensionless parameters

$$
\epsilon=\frac{U}{f L}, \quad \lambda=\frac{\sqrt{g D}}{f L}
$$

are the Rossby number and the nondimensionalized Rossby deformation radius, respectively; the Froude number is $\epsilon / \lambda$. In order to connect the RSW dynamics with its QG limit $(\epsilon \rightarrow 0)$, we utilize the potential vorticity $q$ defined by

$$
1+\epsilon q=\frac{1+\epsilon \zeta}{1+\epsilon \lambda^{-2} \eta-\epsilon b}
$$

Being a rescaled version of Ertel potential vorticity, $q$ is advected by the flow:

$$
\frac{D q}{D t}=0
$$

In these variables, the total energy $\mathcal{H}$ and generalized enstrophy $\mathcal{A}$ take the form

$$
\begin{gathered}
\mathcal{H}=\frac{1}{2} \int\left[\left(1+\epsilon \lambda^{-2} \eta-\epsilon b\right)\left(u^{2}+v^{2}\right)+\lambda^{-2} \eta^{2}\right] d x d y \\
\mathcal{A}=\int\left(1+\epsilon \lambda^{-2} \eta-\epsilon b\right) a(q) d x d y
\end{gathered}
$$


For the purposes of motivating our minimax principle, which distinguishes certain steady solutions of the RSW equations, let us recall the classical analysis of the geostrophic adjustment problem [20]. Consider small amplitude motions governed by the linearized RSW equations, meaning that $|u|,|v|,|\eta| \ll 1$ over the fluid domain, and set $b=0$. This linearized dynamics are reducible to an inhomogeneous Klein-Gordon equation for the height perturbation, namely,

$$
\epsilon^{2} \frac{\partial^{2} \eta}{\partial t^{2}}-\lambda^{2} \triangle \eta+\eta=-\lambda^{2} q
$$

where $q$ is necessarily constant in time according to the linearization of (3.3). The general solution of (3.6) is $\eta=\bar{\eta}+\eta^{\prime}$, where $\bar{\eta}$ corresponds to the steady state and $\eta^{\prime}$ denotes a general solution of the corresponding homogeneous equation. In physical terms, the transient component $\eta^{\prime}$ of this solution is a field of inertia-gravity (Poincaré) waves having the dispersion relation $\omega(k)=\epsilon^{-1} \sqrt{1+\lambda^{2}|k|^{2}}$, where $k$ is the horizontal wave vector. The steady component $\bar{\eta}$ is uniquely determined by the given PV field $q$. More precisely, there is a unique steady state $(\bar{u}, \bar{v}, \bar{\eta})$ defined by

$$
\bar{u}=-\frac{\partial \psi}{\partial y}, \bar{v}=\frac{\partial \psi}{\partial x}, \bar{\eta}=\psi, \quad \text { where } \Delta \psi-\lambda^{-2} \psi=q
$$

along with appropriate boundary conditions on the streamfunction $\psi$. This steady state is geostrophically balanced, and so one can say that from general initial conditions the linearized system relaxes to the balanced state through radiation of the inertia-gravity waves.

The balanced state for linearized RSW dynamics can be characterized as the minimizer of total energy over all fields with given linearized PV, namely, $q=\zeta-\lambda^{-2} \eta$. In this variational principle, the streamfunction $\psi$ arises as the Lagrange multiplier for the PV constraint. The equations of first variation are

$$
\begin{aligned}
0 & =\int\left\{u \delta u+v \delta v+\lambda^{-2} \eta \delta \eta+\psi\left[\delta \zeta-\lambda^{-2} \delta \eta\right]\right\} d x d y \\
& =\int\left\{\left(u+\psi_{x}\right) \delta u+\left(v-\psi_{y}\right) \delta v+\lambda^{-2}(\eta-\psi) \delta \eta\right\} d x d y
\end{aligned}
$$

From this calculation it is evident that the minimizer is the steady geostrophic state $(\bar{u}, \bar{v}, \bar{\eta})$ associated with streamfunction $\psi$.

This principle is emphasized by Vallis [34, 35], who also extends it to finiteamplitude motions. With respect to the exact RSW dynamics he defines a state of "extreme balance" to be a minimizer of $\mathcal{H}$ subject to a pointwise constraint on potential vorticity $q$. His goal is to derive balanced dynamics for the RSW system, in which an approximation to the exact flow evolves through states of extreme balance. Our minimax principle modifies his principle slightly in the sense that we consider minimization of the functional $\mathcal{H}+\theta^{-1} \mathcal{A}$ over all RSW fields with a given $q$, rather than the functional $\mathcal{H}$. For small amplitude motions, the two principles coincide, but the term $\mathcal{A}$ contains an additional order $\epsilon$ contribution for finite-amplitude motions. We include this term so that our minimax principle produces exact steady solutions of finite amplitude.

The potential vorticity constraint in our version of the principle of extreme balance is the linear condition

$$
(1+\epsilon q)\left(1+\epsilon \lambda^{-2} \eta-\epsilon b\right)-(1+\epsilon \zeta)=0
$$


with $q$ given. The objective functional is $\mathcal{L}=\mathcal{A}+\theta \mathcal{H}$, which we maximize over $(u, v, \eta)$ subject to the PV constraint. The parameter $\theta$ is a negative constant, which plays the role of inverse temperature when the enstrophy is interpreted as a negentropy of PV mixing. The negativity of $\theta$ implies the organization of the PV into coherent structures, as in the QG theory $[9,15]$.

We now arrive at our minimax principle, which combines the two principles of extreme balance and minimum enstrophy into a single variational characterization of steady balanced states for the RSW equations. Namely, we solve the variational problem

$$
\min _{q} \max _{(u, v, \eta)}\left\{\mathcal{L}(u, v, \eta):(1+\epsilon q)\left(1+\epsilon \lambda^{-2} \eta-\epsilon b\right)=(1+\epsilon \zeta)\right\} .
$$

As proved in the preceding section, any critical point of $\mathcal{L}$ defines an exact steady solution of the RSW equations. But these minimax critical points have the further property that their PV fields are balanced with respect to geostrophic adjustment. In this sense they represent the extension to finite Rossby number $\epsilon$ of the coherent steady states for QG flow, which minimize $\mathcal{L}_{0}=\mathcal{A}_{0}+\theta \mathcal{H}_{0}$ over $q$.

It is important to clarify the relation between our minimax principle and the notions of balanced dynamics and spontaneous emission of inertia-gravity waves by time-dependent RSW flows. In physical experiments [38], numerical simulations [14, $18]$, and asymptotic theories [19, 39], many investigators have addressed the question of how waves interact with vortical structures. While the phenomenon of adjustment from an initially unbalanced state is relatively well understood, the construction of a time-dependent balanced dynamics is more subtle. This issue becomes relevant to our minimax principle when one interprets the inner maximization as a (nonlinear) projection onto a "slow manifold" of (extreme) balanced states; that is, when one views the maximizer $\left(u^{*}, v^{*}, \eta^{*}\right)$ associated with an arbitrary $q$ as the geostrophically adjusted state relative to $q$. On the one hand, if $q$ itself is not the solution to the outer minimization problem, and therefore is an unsteady state, it could spontaneously generate some inertia-gravity waves. On the other hand, a solution $q$ to the minimax principle as a whole produces an exact steady solution of the RSW equations, which is necessarily free from any inertia-gravity wave emission. Nonetheless, the saddle point nature of the minimax critical points of $\mathcal{A}+\theta \mathcal{H}$ leaves open the possibility that they could be unstable, and hence that the emission of waves could occur during the evolution of an unstable perturbation. The separation of time scales between the vortical and inertia-gravity wave motions suggests, however, that such instabilities would be weak at least for small Rossby numbers.

In contrast to the asymptotic theories of balanced dynamics, our minimax principle pertains to the very long time behavior of solutions - in particular to the effect of finite Rossby number on distinguished steady solutions. These effects lie beyond the time scale of validity of the asymptotic expansions used to decouple fast inertiagravity wave motions from slow vortical modes. Thus, even though we lack a full stability theory, our minimax principle provides quantitative information that is not accessible by other methods.

We draw attention to two points with regard to the the choice of $a(q)$ in the minimax principle. First, even though the generalized enstrophy functional $\mathcal{A}$ can be defined by any continuous convex function $a$, for the sake of definiteness we choose $a$ to be quadratic in the numerical computations that we give below. Second, we include the circulation constraint in the enstrophy expression. In statistical equilibrium theory for QG flows, equilibrium states are characterized as the constrained minimum of a 
generalized convex enstrophy functional subject to fixed energy and circulation [15]. For this reason, we add a linear term to the quadratic $a$ in the minimax principle:

$$
a(q)=\frac{1}{2} q^{2}+\alpha q .
$$

The parameter $\alpha$ is the Lagrange multiplier for the circulation constraint and represents "chemical potential" in the statistical equilibrium interpretation. In all our numerical examples we use this enstrophy expression. Hence our minimax solutions are parameterized by $\epsilon, \lambda, \theta$ and $\alpha$.

\section{Approximate Minimax solutions}

The minimax principle produces exact steady solutions for finite $\epsilon$, even though its formulation is strongly motivated by the separation of RSW dynamics into fast inertiagravity waves and slow vortical modes, which relies on the smallness of $\epsilon$. Accordingly, we could attempt to find minimax critical points directly by invoking an numerical method to solve the nonlinear steady flow equations. Instead we adopt a different approach, which makes explicit the Rossby number-dependence of the approximate solutions. Specifically, we construct approximate solutions of the minimax problem in two steps. First, we solve the inner maximization problem analytically through an asymptotic expansion in Rossby number, thereby obtaining first-order correction to the QG fields. We then insert these first-order expressions into the objective functional and solve the outer minimization problem by a numerical optimization method. The computed solutions therefore have $O\left(\epsilon^{2}\right)$ errors resulting from truncation of the asymptotic expansion, and whatever small numerical errors are associated with the optimization algorithm.

Consider the inner constrained maximization problem in (3.9). Let $\mu(x, y)=$ $(\theta / \epsilon) \psi(x, y)$ denote the Lagrange multiplier for the pointwise PV constraint (3.8); we introduce the scale factor so that $\psi$ is the finite- $\epsilon$ analogue to the geostrophic streamfunction. Then the Lagrange multiplier rule provides the first-order condition:

$$
\begin{aligned}
0= & \delta \int\left(1+\epsilon \lambda^{-2} \eta-\epsilon b\right) a(q)+\frac{\theta}{2}\left[\left(1+\epsilon \lambda^{-2} \eta-\epsilon b\right)\left(u^{2}+v^{2}\right)+\lambda^{-2} \eta^{2}\right] \\
& +\delta \int \frac{\theta}{\epsilon} \psi\left[(1+\epsilon \zeta)-\left(1+\epsilon \lambda^{-2} \eta-\epsilon b\right)(1+\epsilon q)\right] .
\end{aligned}
$$

Calculating the independent variations with respect to $\delta u, \delta v$ and $\delta \eta$, we derive the following equations for the extremal $(u, v, \eta)$ and its multiplier $\psi$ :

$$
\begin{aligned}
\left(1+\epsilon \lambda^{-2} \eta-\epsilon b\right) u & =-\psi_{y}, \\
\left(1+\epsilon \lambda^{-2} \eta-\epsilon b\right) v & =\psi_{x}, \\
\eta+\frac{\epsilon}{2}\left(u^{2}+v^{2}\right)+\epsilon \theta^{-1} a(q) & =\psi(1+\epsilon q) .
\end{aligned}
$$

These three equations together with the PV constraint (3.8) determine the solution of the inner maximization problem.

For small $\epsilon$ we can solve this system up to first order in $\epsilon$ by expanding the unknowns in a power series in $\epsilon$ :

$$
\begin{aligned}
& u(x, y, \epsilon)=u_{0}+\epsilon u_{1}+\epsilon^{2} u_{2}+\ldots \\
& v(x, y, \epsilon)=v_{0}+\epsilon v_{1}+\epsilon^{2} v_{2}+\ldots \\
& \eta(x, y, \epsilon)=\eta_{0}+\epsilon \eta_{1}+\epsilon^{2} \eta_{2}+\ldots \\
& \psi(x, y, \epsilon)=\psi_{0}+\epsilon \psi_{1}+\epsilon^{2} \psi_{2}+\ldots
\end{aligned}
$$


where the coefficients functions are independent of $\epsilon$.

Equating the $\mathrm{O}(1)$ terms yields

$$
\begin{aligned}
u_{0} & =-\psi_{0, y}, \\
v_{0} & =\psi_{0, x}, \\
\eta_{0} & =\psi_{0}, \\
q & =\triangle \psi_{0}-\lambda^{-2} \psi_{0}+b .
\end{aligned}
$$

We immediately see that at the lowest order we recover the geostrophic relations. These $\mathrm{O}(1)$ relations completely determine the field $\left(u_{0}, v_{0}, \eta_{0}\right)$, once the streamfunction is calculated by solving the Helmholtz equation involving the elliptic operator $\triangle-\lambda^{-2}$, which is potential vorticity constraint equation.

Since we are interested in departures from quasi-geostrophy we proceed to the next order in $\epsilon$. Equating the $O(\epsilon)$ terms yields after some calculations:

$$
\begin{aligned}
& u_{1}=-\psi_{1, y}+\lambda^{-2} \psi_{0} \psi_{0, y}-b \psi_{0, y}, \\
& v_{1}=\psi_{1, x}-\lambda^{-2} \psi_{0} \psi_{0, x}+b \psi_{0, x}, \\
& \eta_{1}=\psi_{1}+q \psi_{0}-\frac{1}{2}\left(u_{0}^{2}+v_{0}^{2}\right)-\theta^{-1} a(q)
\end{aligned}
$$

where $\psi_{1}$ is determined using the potential vorticity constraint through

$$
\begin{gathered}
\triangle \psi_{1}-\lambda^{-2} \psi_{1}=\lambda^{-2}\left[\frac{1}{2}\left|\nabla \psi_{0}\right|^{2}+3 \psi_{0} \triangle \psi_{0}-2 \lambda^{-2} \psi_{0}^{2}-\theta^{-1} a(q)\right] \\
+3 \lambda^{-2} b \psi_{0}-2 b \triangle \psi_{0}-\nabla b \cdot \nabla \psi_{0}-b^{2} .
\end{gathered}
$$

With the appropriate boundary conditions, we find $\psi_{1}$ by solving this Helmholtz equation. Given $\psi_{1}$, the $O(\epsilon)$ field $\left(u_{1}, v_{1}, \eta_{1}\right)$ is determined.

We now substitute the first-order solution of the inner maximization problem into the objective functional, and thereby we generate the approximate objective functional for the outer minimization problem. A lengthy calculation produces $\mathcal{H}$ and $\mathcal{A}$ to the first order in $\epsilon$, namely,

$$
\begin{aligned}
\mathcal{H}_{\epsilon}(q)= & \frac{1}{2} \int\left|\nabla \psi_{0}\right|^{2}+\lambda^{-2} \psi_{0}^{2} \\
& +\epsilon \int\left[\frac{5}{2} \lambda^{-2} \psi_{0}\left|\nabla \psi_{0}\right|^{2}+\lambda^{-4} \psi_{0}^{3}-\frac{1}{2} b\left|\nabla \psi_{0}\right|^{2}-2 \lambda^{-2} b \psi_{0}^{2}+b \psi_{0} \triangle \psi_{0}+\psi_{0} b^{2}\right] \\
\mathcal{A}_{\epsilon}(q)= & \int a(q)+\epsilon \int\left(\lambda^{-2} \psi_{0}-b\right) a(q) .
\end{aligned}
$$

In these integrals, $\psi_{0}=\left(\triangle-\lambda^{-2}\right)^{-1}(q-b)$.

The minimax principle can thus be reduced to the single minimization problem

$$
\min _{q} \mathcal{L}_{\epsilon}=\mathcal{A}_{\epsilon}+\theta \mathcal{H}_{\epsilon} .
$$

While a solution of the minimax principle (3.9) is an exact steady state of the RSW dynamics, a solution of (4.6) is an approximate steady state accurate up to $O(\epsilon)$. These approximate solutions are the first-order corrections to the coherent states obtained in the analogous QG theory. Moreover, this defining variational principle 
for small $\epsilon$ has exactly the same form as the corresponding QG variational principle, which it tends to as $\epsilon \rightarrow 0$.

The outer unconstrained minimization problem is solved numerically using a nonlinear optimization method. Specifically, in our computations of one-dimensional solutions we use a well-known quasi-Newton method, namely, the Broyden-FletcherGoldfarb-Shanno algorithm [21].

\section{Steady balanced shear flows}

In this section we present some solutions of the minimax principle in the onedimensional case. That is, we consider zonal shear flows $u=u(y), v=0, \eta=\eta(y)$ in a channel $0<y<1$ with flat bottom topography $b=0$. As mentioned above, we use the enstrophy function $a(q)$ given by (3.10). These numerical experiments provide some insight into first-order Rossby number effects in the simplest setting relevant to geophysical problems.

In the displayed computations, we consider a range of values of the parameters $\epsilon$ and $\lambda$ chosen to be representative of the physical values in the atmosphere and the oceans. We vary the Rossby number $\epsilon$ from 0 to 0.3 and we consider values of the normalized deformation radius $\lambda$ from 0.1 to $1[6,10,27]$.

In our numerical experiments, we first compute the quasi-geostrophic states, which are solutions to the minimization problem when $\epsilon=0$. Then we construct a branch of approximate solutions with finite $\epsilon$ using a continuation strategy. Namely, we begin with very small $\epsilon$ and compute solutions with increasing $\epsilon$, retaining the solution for $\epsilon_{1}$ as an initial guess in the optimization algorithm for finding the solution with a slightly larger $\epsilon_{2}$, and so forth.

In the special case that we consider in this section, the QG states are explicit, being the solutions to a linear boundary-value problem on the interval $0<y<1$. These solutions are determined by minimizing the following functional of $q$,

$$
\int_{0}^{1}\left[\frac{1}{2} q^{2}+\alpha q+\frac{\theta}{2}\left(\left[\frac{d \psi_{0}}{d y}\right]^{2}+\lambda^{-2} \psi_{0}^{2}\right)\right] d y
$$

which implies that they satisfy

$$
q=\frac{d^{2} \psi_{0}}{d y^{2}}-\lambda^{-2} \psi_{0}=\theta \psi_{0}-\alpha, \quad \psi_{0}(0)=0=\psi_{0}(1)
$$

The boundary conditions on the streamfunction impose zero net flux through the channel. These conditions reflect the fact that the linear impulse invariant is not included in the variational principle; inclusion of the linear impulse produces a nonzero translational speed, which can be included into the steady shear flow giving it a nonzero net flux.

There are two cases. When $\lambda^{-2}+\theta>0$, the geostrophic streamfunction is

$$
\psi_{0}(y)=\frac{\alpha}{\kappa^{2}}\left[1-\frac{\cosh \kappa(y-1 / 2)}{\cosh \kappa / 2}\right] \quad \text { with } \kappa^{2}=\lambda^{-2}+\theta .
$$

When $\lambda^{-2}+\theta<0$,

$$
\psi_{0}(y)=-\frac{\alpha}{\kappa^{2}}\left[1-\frac{\cos \kappa(y-1 / 2)}{\cos \kappa / 2}\right] \text { with } \kappa^{2}=-\left(\lambda^{-2}+\theta\right) .
$$

In the second case, the solution exists provided that $\kappa$ is not an integral multiple of $\pi$. The physical significance of the parameter $\alpha$ is revealed by these solutions: when $\alpha<0$, 


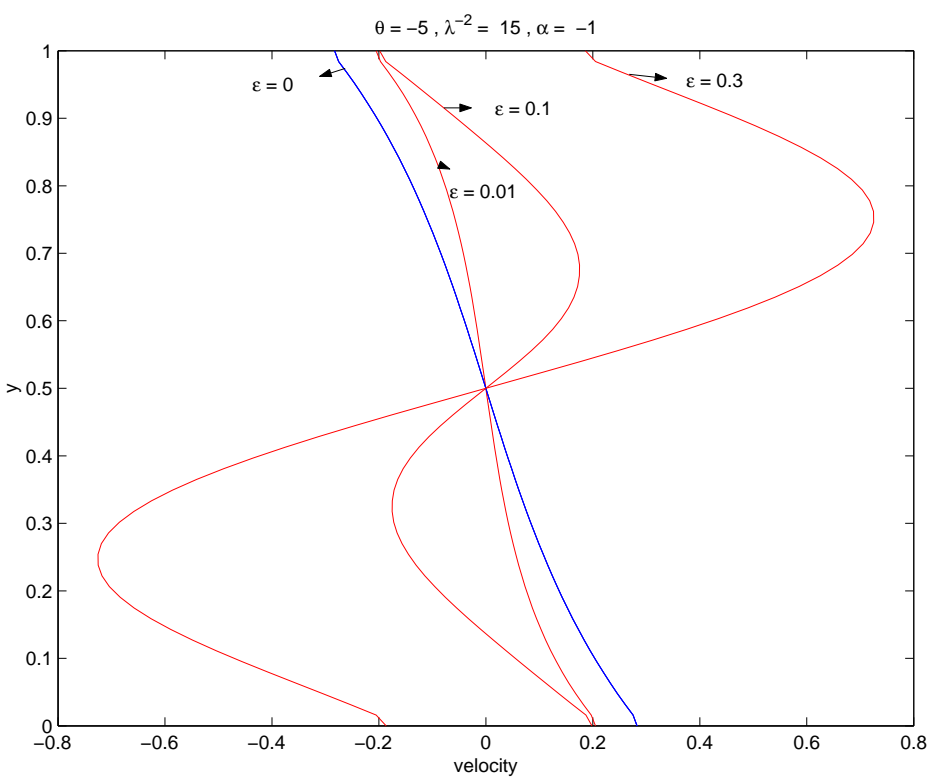

FIG. 5.1. The flows with increasing $\epsilon$ for fixed parameters $\theta=-5, \alpha=-1, \lambda^{-2}=15$. As the Rossby number $\epsilon$ varies from 0 to 0.3 , a weak cyclonic shear develops into a strong anticyclonic band.

the shear $u_{0}=-d \psi_{0} / d y$ is cyclonic (meaning that the circulation is positive), and when $\alpha>0$ it is anticyclonic (the circulation is negative); we assume that $\lambda^{-2}+\theta>-\pi^{2}$.

In our computations we choose either $\alpha=-1$ (cyclonic shear) or $\alpha=+1$ (anticyclonic shear). We vary $\theta$ to cover both of the above cases, including the critical case when $\theta$ approaches $-\lambda^{-2}-\pi^{2}$.

In figure 5.1 we display a typical branch of solutions to the minimax principle with increasing Rossby number $\epsilon$. In this set of computed solutions the deformation radius is relatively small compared to the unit channel width, having $\lambda^{-2}=15$. The breakdown of the QG solution, which is a weak cyclonic shear, is therefore anticipated even for fairly small $\epsilon$. Indeed, for $\epsilon=0.1$, which is typical of many geophysical flow regimes, the RSW flow is qualitatively different from the QG flow, in that it develops an interior band of anticyclonic shear. The magnitude of the shear in this anticyclonic band increases as $\epsilon$ is increased; the figure shows the solution for $\epsilon=0.3$, which may be at the limit of the validity of the asymptotics.

In figure 5.2, we examine the analogous effect, but now beginning with a QG flow that is an anticyclonic shear flow. The normalized deformation radius is now $\lambda=0.2$, which emulates a regime of weak stratification. In this regime the minimax solutions depend less strongly on the Rossby number, and they retain the general anticyclonic features of the QG flow for small $\epsilon$. Nonetheless, when the Rossby number is increased to $\epsilon=0.2$, the minimax solution forms two cyclonic bands surrounding a band of nearly uniform flow. In the anticyclonic case the qualitative features of the QG flow field are substantially changed only for relatively large values $\epsilon$.

The breakdown of symmetry between cyclones and anticyclones is an important feature of RSW turbulence, which distinguishes it from QG turbulence. This phenomenon has been investigated by a number of studies $[6,8,22,28]$. A plausible 


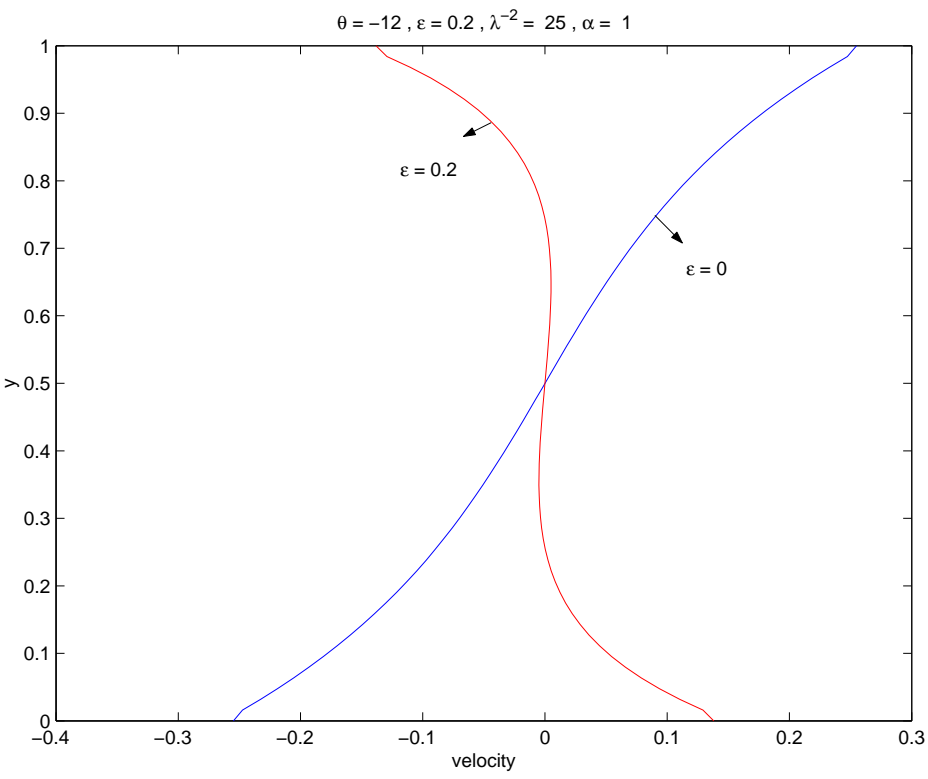

FIG. 5.2. An anticyclonic $Q G$ shear becomes cyclonic when the Rossby number is increased to $\epsilon=0.2$

physical argument for the cyclone-anticyclone asymmetry is given by Polvani et al. [28]. They note that cyclones correspond to the depression in the fluid layer, which implies that the local value for the deformation is smaller than that of anticyclones. This restricts the interaction of the small cyclonic eddies and thus inhibits their growth into larger cyclones through merger. On the other hand, the anticyclones correspond to expansion of the fluid layer, making the local radius of deformation larger, and resulting in stronger interaction and hence an increased likelihood of merger. Consequently, the distribution of coherent vortices in decaying RSW turbulence is skewed toward the anticyclonic. This phenomenology is supported by our computations of steady balanced flows, in that we observe finite- $\epsilon$ effects that preferentially enhance anticyclonic shear flows. While the $O(\epsilon)$ corrections can create cyclonic structures as in figure 5.2, these flows are typically weaker than the anticyclonic structures, such as those displayed in figure 5.1.

In the solutions displayed in figures 5.1 and 5.2, the "inverse temperature" $\theta$ is chosen in the range $\theta+\lambda^{-2}>-\pi^{2}$. For such $\theta$, the Lyapunov functional $\mathcal{A}_{0}+\theta \mathcal{H}_{0}$ is positive-definite on the QG equilibrium state, and consequently the Arnold second stability criterion is satisfied with respect to QG dynamics. It is interesting to consider values of $\theta$ that are near or slightly beyond this critical value, especially since some important geophysical shear flows are known to lie in this transitional regime. In particular, Dowling has shown that the midlatitude zonal jet structure that is the most prominent and permanent feature of Jupiter's weather layer lies in this regime [11]. In figure 5.3, we therefore take a negative $\theta$ lying beyond the critical value for the Arnold sufficient condition for stability for QG flow. (We emphasize that the fact that this stability condition is not satisfied does not necessarily imply that the QG flows are unstable. In fact, refined stability theorems that utilize the independent conservation of both $A_{0}$ and $\mathcal{H}_{0}$ provide stability for minimum negentropy states 


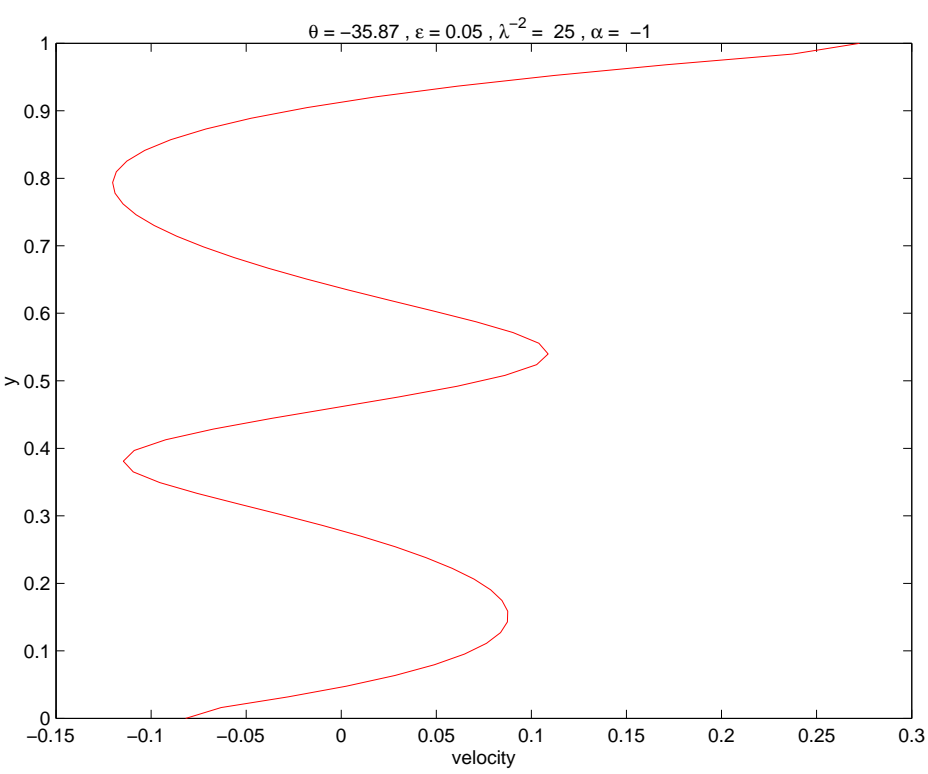

FIG. 5.3. The emergence of bands on the scale of the Rossby deformation radius. The parameters $\epsilon=0.05, \lambda=0.2$ are taken to represent a mid-latitude band in Jupiter, and $\theta$ is chosen to be slightly beyond the quasi-geostrophic stability limit based on the Arnold criterion.

subject to constrained energy and circulation in this QG regime [15, 16]. Also, we stress that these stability criteria apply to QG dynamics, not to finite-Rossby number RSW dynamics.) At finite $\epsilon$, steady balanced solutions continue to exist beyond the QG critical value, and such a solution is displayed in figure 5.3. The key qualitative feature of this shear flow is the reversing jet structure which emerges on the scale of the deformation radius $\lambda=0.2$. Thus, the minimax principle is able to reproduce prototypical flow structures that resemble Jupiter's mean zonal winds, even in this simple situation without bottom topography. However, the stability of any of these approximate RSW flows is an open question for finite $\epsilon$.

These numerical results show that the finite-Rossby number effect included in our steady, balanced RSW flows is quantitatively significant, especially in the regime of small deformation scale. This conclusion is consistent with some inferences from physical data. Indeed, quasigeostrophy is believed to break down at small deformation scales [4], and by analyzing the wind data in the band containing the Great Red Spot of Jupiter, Dowling and Ingersoll $[12,13]$ estimated that the QG approximation makes a 30 percent error.

\section{Shear flows over bottom topography}

As another illustration of the minimax principle, we now consider finite-Rossby number corrections to a $1+1 / 2$-layer model of Jupiter's atmosphere [10]. In this model, the upper layer is shallow and active, and it overlays a lower layer that is deeper, denser and passive. The deep lower layer represents a neutrally stratified deep atmosphere, the structure of which is only inferred from observations of the upper layer. The upper layer is dynamically equivalent to a single shallow layer with a bottom topography that includes the effect of the deep lower flow and the $\beta$ effect [10]. A detailed analysis of the effective RSW dynamics of observed eddies 
has demonstrated that there exists a strong zonal shear flow in the lower layer [11]. This effective bottom topography has been included in QG models of the upper layer zonal shears and embedded vortices based on the statistical equilibrium theory $[4,33]$. In terms of the geostrophic streamfunction for the lower layer $\psi_{2}(y)$ and the scaled Coriolis gradient $\beta$, the effective bottom topography is $b(y)=\lambda^{-2} \psi_{2}+\beta y$.

We compute minimax RSW solutions for exactly the same formulation and parameter choices used in the statistical equilibrium QG model in [33] (see also [25]). In this way we quantify the departures from quasi-geostrophy in this specific physical situation. Namely, we consider two midlatitude bands on Jupiter, for which the latitude $\Theta$ lies in the range $\Theta_{-}<\Theta<\Theta_{+}$. Each band is selected to contain two belts (cyclonic regions) and two zones (anticyclonic regions). The southern hemisphere band lies between $36.6^{\circ} \mathrm{S}$ and $13.7^{\circ} \mathrm{S}$; the northern hemisphere band lies between $23.1^{\circ} \mathrm{N}$ to $42.5^{\circ} \mathrm{N}$. (We refer both these domains to northern hemisphere sign conventions to make them isomorphic problems.)

The characteristic length scale $L$ is defined to be half the channel width, $L=\left(\Theta_{-}-\right.$ $\left.\Theta_{+}\right) r_{0} / 2$, where $r_{0}=7 \times 10^{7} \mathrm{~m}$ represents the radius of Jupiter. The characteristic velocity scale $U$ is defined as the RMS velocity of the centered Limaye profile for the permanent zonally averaged winds in the upper layer [24].

The nondimensionalized problem is posed on the domain $-1<y<+1$, and its dimensionless groups are

$$
\epsilon=\frac{U}{f\left(\Theta_{0}\right) L}, \lambda=\frac{c}{f\left(\Theta_{0}\right) L}, \quad \beta=\frac{f^{\prime}\left(\Theta_{0}\right) L^{2}}{r_{0} U}
$$

where the Coriolis parameter $f(\Theta)=2 \Omega \sin \Theta$, with the angular speed of rotation $\Omega=$ $1.76 \times 10^{-4} s^{-1}$, is evaluated at the central latitude $\Theta_{0}=\left(\Theta_{+}+\Theta_{-}\right) / 2$, and $c=454 s^{-1}$ is the estimated gravity wave speed for Jupiter [11]. The Rossby number is found to be $\epsilon \approx 0.014$ for the southern hemisphere, and $\epsilon \approx 0.021$ for the northern hemisphere. The normalized deformation scale is roughly $\lambda \approx 0.2$ in both the northern and southern bands. Thus, our problem lies in a range of parameters for which substantial departure from quasi-geostrophy may be expected.

Following Dowling [11], the streamfunction $\psi_{2}$ for the lower layer is inferred from the observed zonally-averaged cloud-top Limaye profile in the upper layer $\tilde{u}$. That is, $\psi_{2}$ is determined so that the streamfunction $\tilde{\psi}$ of the observed zonal shear coincides with the minimum enstrophy state subject to fixed energy and circulation. Specifically, the QG mean-field equation

$$
\frac{d^{2} \tilde{\psi}}{d y^{2}}-\lambda^{-2} \tilde{\psi}+\beta y+\lambda^{-2} \psi_{2}=\theta \tilde{\psi}-\alpha
$$

where $\theta$ and $\alpha$ are the multipliers corresponding to the constraints on energy and circulation, respectively, defines the profile $\psi_{2}(y)$. But the values of $\theta$ and $\alpha$ are not known a priori, and so a selection procedure is needed to determine them. For any $\theta$, we set $\alpha$ so that $\int_{-1}^{1} \psi_{2} d y=0$, which centers the lower layer flow. We choose $\theta=$ $-\lambda^{-2}-(\pi / 2)^{2}$, the first eigenvalue of the operator $\left(\triangle-\lambda^{-2}\right)$ in the band $-1<y<1$. This choice puts the QG equilbrium state at the critical value with respect to Arnold's second stability criterion, in agreement with Dowling's analysis of observations.

We find strikingly different results in the two bands when we compute the $O(\epsilon)$ corrections to the QG predictions. In the southern hemisphere the RSW shear flow provided by the minimax principle is substantially different from the QG equilibrium 


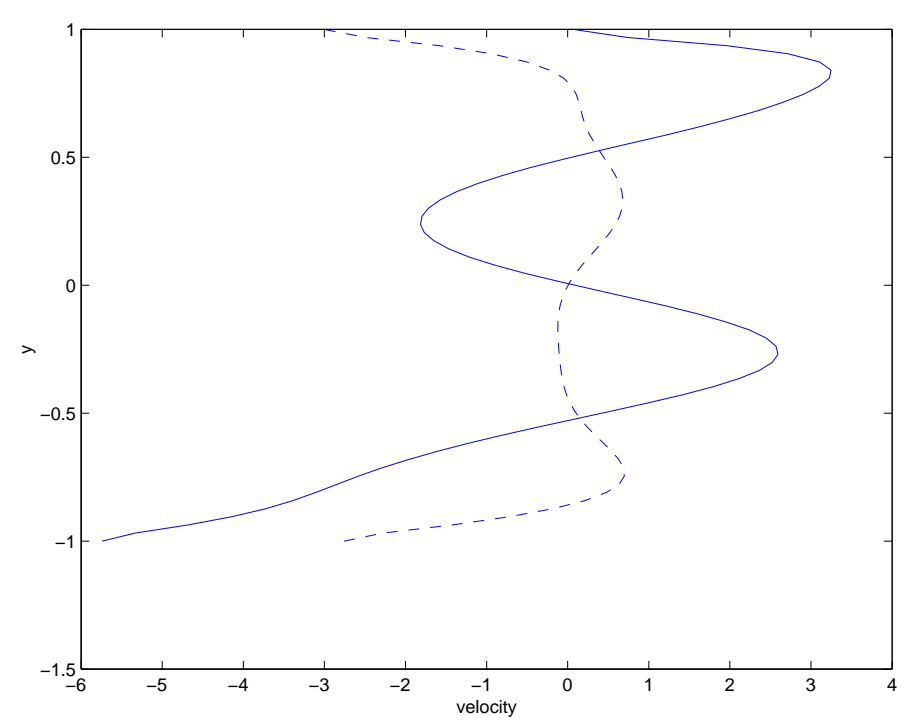

FIG. 6.1. Zonal shear flow with $\epsilon \approx 0$ (dashed) and $R S W$ shear with $\epsilon=0.014$ (solid) in Southern Hemisphere band from $36.6^{\circ} S$ to $13.7^{\circ} S$. The $Q G$ flow changes substantially when $O(\epsilon)$ correction is added.

state at $\epsilon \approx 0.014$. These two zonally-averaged shear profiles are displayed in figure 6.1. The finite- $\epsilon$ shear is much stronger than the QG state and its peak velocities are much larger. By contrast, the QG flows in the northern hemisphere are much more robust with respect to the finite- $\epsilon$ correction. Figure 6.2 shows that there is only a small difference between the QG shear flow and the corresponding RSW flow at $\epsilon \approx 0.021$.

In two-dimensional QG computations of most probable states [33], there is also a qualitative difference between the northern and southern hemisphere bands. In that model, the effects of the thermal forcing of the weather layer by convection is parametrized by the skewness of the distributions of fine-grained potential vorticity. When the skewness is increased toward the anticyclonic the shear flow in the southern band transitions to a Great Red Spot vortex, centered around $23^{\circ} S$, whereas the flow in the northern band remains a zonal shear. In light of the fact that the large coherent structures in Jupiter's atmosphere are energized by small-scale convection, this provides a plausible explanation of the formation of the GRS.

In our RSW model of steady balanced states, we find that there is a natural cyclone-anticyclone asymmetry, and so the formation of coherent anticyclonic vortices, such as the GRS, may be partially attributed to the free dynamics at finite Rossby number and are not entirely dependent upon the nature of the forcing. Indeed, we find that the shear flows in the northern band are well predicted by QG theory, but that the zonally averaged shears in the southern band are not. Given the tendency for the $O(\epsilon)$ corrections to QG to favor anticyclonic structures, it is reasonable to conjecture that a two-dimensional computation of steady balanced RSW states might predict the formation of a large anticyclone in the southern band. We leave this interesting task to further research, since our numerical method is implemented only for onedimensional solutions of the minimax principle. Finally, we remark that possibly 


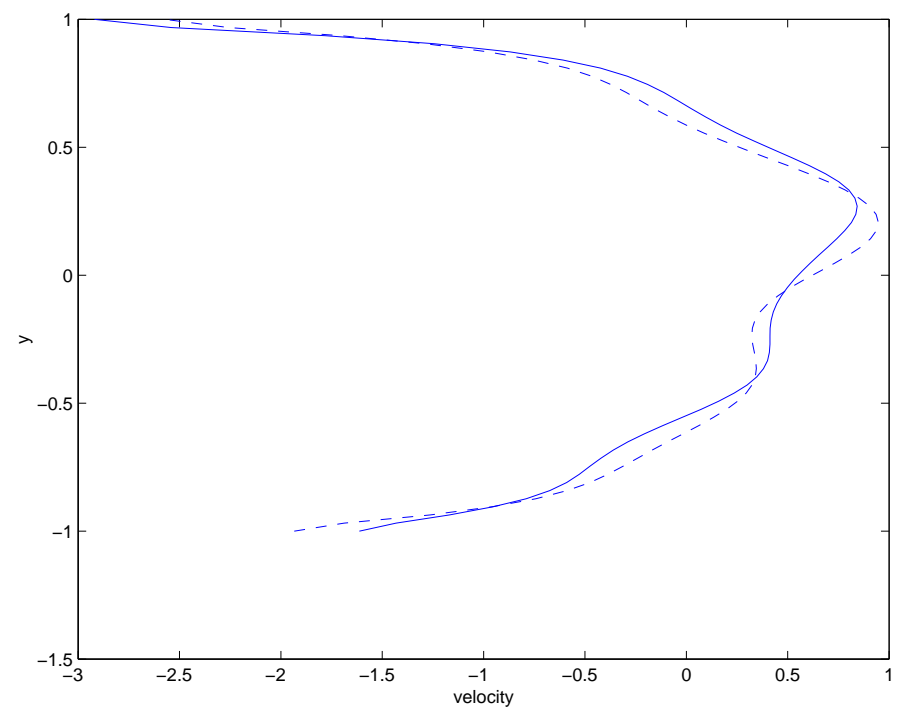

FIG. 6.2. Zonal shear flow with $\epsilon \approx 0$ (dashed) and $\epsilon=0.021$ (solid) in Northern Hemisphere band from $23.1^{\circ} \mathrm{N}$ to $42.5^{\circ} \mathrm{N}$. There is little change when $O(\epsilon)$ correction is added.

both the anticyclonic skewness of the the PV fluctuations due to thermal convection and the cyclonic-anticyclonic asymmetry due to finite Rossby number dynamics may be mechanisms that cause the preferential formation of coherent anticyclonic vortices in the weather layer. Quantifying the relative importance of each of these effects is another interesting question for future investigations.

Acknowledgement. This paper is dedicated with great admiration to Andy Majda on the occasion of his sixtieth birthday. This research was supported in part by grants from the National Science Foundation (NSF-DMS-0207064, NSF-DMS0604071).

\section{REFERENCES}

[1] J.S. Allen, J.A. Barth and P.A. Newberger, On intermediate models for barotropic continental shelf and slope flow fields: part I, J. Phys. Oceanogr., 20, 1017-1042, 1990.

[2] J.S. Allen, J.A. Barth and P.A. Newberger, On intermediate models for barotropic continental shelf and slope flow fields: part II J. Phys. Oceanogr., 20, 1949-1973, 1990.

[3] W. Blumen, Geostrophic adjustment, Rev. Geophysics and Space Physics, 10, 485-528, 1972.

[4] F. Bouchet and J. Sommeria, Emergence of intense jets and Jupiter's Great Red Spot as maximum-entropy structures, J. Fluid Mech., 464, 165-207, 2002.

[5] F.P. Bretherton and D.B. Haidvogel, Two dimensional turbulence over topography, J. Fluid Mech., 78, 129-154, 1976.

[6] J.G. Charney and G.R. Flierl, Oceanic analogies of large scale atmospheric motions, in Evolution of Physical Oceanography, MIT Press, Cambridge, 504-548, 1981.

[7] P.H. Chavanis and J. Sommeria, Statistical mechanics of the shallow water system, Phys. Rev. E, 65, 026302, 2002.

[8] B. Cushman-Roisin and B. Tang, Geostrophic turbulence and emergence of eddies beyond the radius of deformation, J. Phys. Oceanogr., 20, 97-113, 1990.

[9] M.T. DiBattista, A.J. Majda and B. Turkington, Prototype geophysical vortex structures via large-scale statistical theory, Geophys. Astrophys. Fluid Dyn., 89, 235-283, 1998. 
[10] T.E. Dowling, Dynamics of Jovian atmospheres, Annual Rev. Fluid Mech., 27, 293-334, 1995.

[11] T.E. Dowling, Estimate of Jupiter's deep zonal-wind profile from Shoemaker-Levy 9 data and Arnol'd's second stability criterion, Icarus, 117, 439-442, 1995.

[12] T. Dowling and A.P. Ingersoll, Potential vorticity and layer thickness variations in the flow around Jupiter's Great Spot and White Oval BC, J. Atmos. Sci., 46, 1380-1396, 1988.

[13] T. Dowling and A.P. Ingersoll, Jupiter's Great Red Spot as a shallow water system, J. Atmos. Sci., 45, 3254-3278, 1989.

[14] D.G. Dritschel and A. Viudez, The persistence of balance in geophysical flows, J. Fluid Mech., $570,365-383,2007$.

[15] R.S. Ellis, K. Haven and B. Turkington, Nonequivalent statistical equilibrium ensembles and refined stability theorems for most probable flows, Nonlinearity, 15, 239-255, 2002.

[16] R.S. Ellis, K. Haven and B. Turkington, Analysis of statistical equilibrium models of geostrophic turbulence, J. Appl. Math. Stochastic Anal., 15, 341-361, 2002.

[17] M. Farge and R. Sadourny, Wave vortex dynamics in rotating shallow water, J. Fluid Mech., 206, 433-462, 1989.

[18] R. Ford, Gravity wave radiation from vortex trains in rotating shallow water, J. Fluid Mech., 281, 81-118, 1994.

[19] R. Ford, M.E. McIntyre and W.A. Norton, Balance and the slow quasimanifold: some explicit results, J. Atmos. Sci., 57, 1236-1254, 2000.

[20] A.E. Gill, Atmosphere-Ocean Dyamics, Academic Press, 1982.

[21] P.E Gill, W. Murray and M. H Wright, Practical Optimization, New York Academic Press, 1981.

[22] A.C. Kuo and L.M. Polvani, Nonlinear geostrophic adjustment, cyclone/anticyclone asymmetry, and potential vorticity rearrangement, Phys. of Fluids, 12, 1087-1100, 2000.

[23] C.E. Leith, Minimum enstrophy vortices, Phys. of Fluids, 27, 1388-1395, 1984.

[24] S.S. Limaye, Jupiter: New estimates of the mean zonal flow at the cloud level, Icarus, 65, $332-352,1986$.

[25] A.J. Majda and X. Wang, Nonlinear Dynamics and Statistical Theories for Basic Geophysical Flows, Cambridge Univ. Press, 2006.

[26] A.J. Majda and M. Holen, Dissipation, topography, and statistical theories for large scale coherent structure, Commun. Pure Appl. Math., 50, 1183-1234, 1997.

[27] D.B. Olson, Rings in the ocean, Annual Rev. Earth Planet Sci., 19, 283-311, 1991.

[28] L.M. Polvani, J.C. McWilliams, M.A. Spall and R. Ford, The coherent structures of shallow water turbulence: deformation-radius effects, cyclone/anticyclone asymmetry and gravity wave generation, Chaos, 4, 177-186, 1994.

[29] J. Pedlosky, Geophysical Fluid Dynamics, Second edition, Springer-Verlag, 1987.

[30] R. Salmon, G. Holloway and M. Hendershott, The equilibrium statistical mechanics of simple quasi-geostrophic models, J. Fluid Mech., 75, 691-703, 1976.

[31] T.G. Shepherd, Symmetries, conservation laws, and Hamiltonian structure in geophysical fluid dynamics, Adv. Geophysics, 32, 287-338, 1990.

[32] B. Turkington, Statistical equilibrium measures and coherent states in two-dimensional turbulence, Commun. Pure Appl. Math., 52, 781-809, 1999.

[33] B. Turkington, A.J. Majda, K. Haven and M. DiBattista, Statistical equilibrium predictions of jets and spots on Jupiter, Proc. Nat. Acad. Sci. USA, 98, 12346-12350, 2001.

[34] G.K. Vallis, Mechanisms and parameterizations of geostrophic adjustment and a variational approach to balanced flow, J. Atmos. Sci., 49, 1144-1160, 1992.

[35] G.K. Vallis, Atmospheric and Oceanic Fluid Dynamics: Fundamentals and Large-Scale Circulation, Cambridge Univ. Press, 2006.

[36] T. Warn, Statistical mechanical equilibria of the shallow water equations, Tellus, 38A, 1-11, 1986 .

[37] P.B. Weichman and D.M. Petrich, Statistical equilibrium solutions of the shallow water equations, Phys. Rev. Lett., 86, 1761-1764, 2001.

[38] P.D. Williams, T.W.N. Haine and P.L. Read, Inertia-gravity waves emitted from balanced flow: observations, properties, and consequences, J. Atmos. Sci., 65, 3543-3556, 2008.

[39] V. Zeitlin, Decoupling of balanced and unbalanced motions and inertia-gravity wave emission: small versus large Rossby numbers, J. Atmos. Sci., 65, 3528-3542, 2008. 